\title{
The Relationship Between The Apparent Diffusion Coefficient and The Ki-67 Proliferation Index in Intracranial Solitary Fibrous Tumor/Hemangiopericytoma
}

Shenglin Li ( $\sim$ lishl18@lzu.edu.cn ) Lanzhou University Second Hospital https://orcid.org/0000-0003-4325-6009

Qing Zhou

Lanzhou University Second Hospital

Peng Zhang

Lanzhou University Second Hospital

Shize Ma

Lanzhou University Second Hospital

Caiqiang Xue

Lanzhou University Second Hospital

Juan Deng

Lanzhou University Second Hospital

Xianwang Liu

Lanzhou University Second Hospital

Junlin Zhou

Lanzhou University Second Hospital https://orcid.org/0000-0001-8109-6347

\section{Research Article}

Keywords: Intracranial, Solitary fibrous tumor/hemangiopericytoma, Ki-67 proliferation index, MRI, Apparent diffusion coefficient

Posted Date: September 30th, 2021

DOl: https://doi.org/10.21203/rs.3.rs-903739/v1

License: (c) (i) This work is licensed under a Creative Commons Attribution 4.0 International License. Read Full License 
Version of Record: A version of this preprint was published at Neurosurgical Review on November 11th, 2021. See the published version at https://doi.org/10.1007/s10143-021-01687-y. 


\section{Abstract \\ Objiective:}

This study evaluated the value of the apparent diffusion coefficient (ADC) in distinguishing grade II and III intracranial solitary fibrous tumors /hemangiopericytomas and explored the correlation between ADC and Ki-67.

\section{Methods}

The preoperative MRIs of 37 patients treated for solitary fibrous tumor/hemangiopericytoma (grade II, $\mathrm{n}=$ 15 and grade III, $n=22$ ) in our hospital from 2011 to October 2020 were retrospectively analyzed. We compared the difference between the minimum, average, maximum, and relative ADCs based on tumor grade and examined the correlation between $A D C$ and Ki-67. Receiver operating characteristic curve analysis was used to analyze the diagnostic efficiency of the ADC.

\section{Results}

There were significant differences in the average, minimum, and relative ADCs between grade II and III patients. The optimal cutoff value for the relative ADC value to differentiate grade II and III tumors was 0.998 , which yielded an area under the curve of 0.879 . The Ki- 67 proliferation indexes of grade II and III tumors were significantly different, and the average $(r=-0.427)$, minimum $(r=-0.356)$, and relative $(r=$ $-0.529)$ ADCs were significantly negatively correlated with the Ki-67 proliferation index.

\section{Conclusions}

ADC can be used to differentiate grade II and III intracranial solitary fibrous tumors/hemangiopericytomas. Our results can be used to formulate a personalized surgical treatment plan before surgery.

\section{Introduction}

The 2016 World Health Organization CNS tumor classification indicates that solitary fibrous tumors (SFTs) and hemangiopericytomas (HPCs) both exhibit the 12q13 chromosome inversion and expression the NAB2-STAT6 fusion product. Given the partial overlap between SFTs and HPCs in pathology, such lesions are currently described as SFT/HPC. In addition, to better interpret the pathological and clinical features of SFT/HPC, SFT/HPC is divided into three grades: grade I SFT/HPC has benign characteristics and a good prognosis, whereas grade II and III SFT/HPC are malignant tumors prone to infiltration of the surrounding tissue structure, postoperative tumor recurrence, and extracranial metastasis $[1,4,14]$. However, even though grade II and III SFT/HPC are malignant tumors derived from meningeal interstitial 
capillary Zimmerman cells, they are associated with different outcomes. Grade III SFT/HPC is more prone to recurrence and metastasis, and the overall survival and disease-free progression intervals are significantly shorter than those of grade II SFT/HPC $[1,4,15]$.

Previous studies have shown that radiological features can indirectly reflect the pathological grade of intracranial SFT/HPC. For instance, necrosis, hemorrhage, blurred tumor-brain interface, and obvious brain edema all indicate III grade SFT/HPC [16-18]. However, these radiological features are subjective and cannot quantitatively evaluate molecular information about the tumor. DWI, a non-invasive technique for reflecting the diffusion of water molecules in living tissues, has been widely used in various fields of tumor quantitative analysis. Onishi et al [19]. found that the minimum ADC (MinADC) could distinguish between tumors with high and low Ki-67 expression in patients with mucinous breast cancer, and the MinADC had a strong negative correlation with the Ki-67 proliferation index $(r=-0.825, P<.0001)$. Because grade III SFT/HPC often has higher cell density and mitotic activity, we speculate that the ADC may be able to differentiate grade II and III intracranial SFT/HPC, and there may be a relationship between the ADC and the Ki-67 proliferation index [27]. As far as we know, there is no study that uses only $A D C$ values to differentiate grade II and III intracranial SFT/HPC or evaluates the relationship between $A D C$ and the Ki-67 proliferation index. Therefore, the purposes of this study were to examine the ability of the preoperative ADC to distinguish grade II and III intracranial SFT/HPC and to study the relationship between the $A D C$ and the Ki-67 proliferation index.

\section{Methods}

\section{Patients}

The study was approved by the ethics committee of our agency. Because this was a retrospective study, the requirement for informed consent was waived. The preoperative MRI and postoperative pathological data of 42 patients with pathologically confirmed grade II and III intracranial SFT/HPC treated in our hospital from January 2011 to October 2020 were analyzed retrospectively. The inclusion criteria were as followed: (1) complete preoperative MRI data (three patients were excluded); (2) complete pathological data after the first operation; and (3) no tumor-related treatment before surgery (two patients were excluded: preoperative vascular interventional embolization). Finally, 37 patients with intracranial SFT/HPC were included, including 15 patients with grade II disease and 22 patients with grade III disease.

\section{Mri Protocol}

Using the Siemens Verio 3.0T (Germany) superconducting MR scanner, the plain scan sequence included axial/sagittal SE-T1WI (ITR $550 \mathrm{~ms}$, TE $12 \mathrm{~ms}$ ), axial FSE-T2WI (TR $2200 \mathrm{~ms}$, TE $90 \mathrm{~ms}$ ), and FLAIR T2WI (TR $9000 \mathrm{~ms}$, TE $110 \mathrm{~ms}$, TI $2371 \mathrm{~ms}$ ), with an FOV $320 \mathrm{~mm} \times 320 \mathrm{~mm}$, matrix $256 \times$ 256, axial layer thickness $5 \mathrm{~mm}$, layer spacing $1.5 \mathrm{~mm}$, sagittal layer thickness $8 \mathrm{~mm}$, and layer spacing $2 \mathrm{~mm}$. DWI used the SE-EPI sequence plus frequency selective fat suppression technology, TR $4000 \mathrm{~ms}$, TE $100 \mathrm{~ms}$, layer 
thickness $9 \mathrm{~mm}$, layer spacing $1 \mathrm{~mm}$, FOV $260 \mathrm{~mm} \times 260 \mathrm{~mm}$, and matrix $256 \times 192$. Diffusion gradients ( $b$ value $=0,1000 \mathrm{~s} / \mathrm{mm}^{2}$ ) were applied in the directions of the $\mathrm{x}, \mathrm{y}$, and $\mathrm{z}$ axes. The contrast agent was $0.1 \mathrm{mmol} / \mathrm{kg}$ Gd-DTPA, the flow rate was $3 \mathrm{~mL} / \mathrm{s}$, and the axial-, sagittal-, and coronal-enhanced T1WI were obtained. All patients received T1WI, T2WI, T2-FLAIR, DWI, and MRI enhanced scans before treatment.

\section{Image Analysis}

Two radiologists with more than 10 years of experience in CNS diagnosis analyzed the scans in a Singleblind manner (the pathological diagnosis of SFT/HPC was known, but the grade of SFT/HPC was not clear). The measured data included tumor size, tumor volume, maximum ADC, average ADC (MeanADC), MinADC, and relative ADC (rADC). The average of the two observers was taken as the result.

All tumor specimens were stained with H\&E. The immunohistochemical analysis of Ki-67 was performed using a monoclonal mouse anti-human Ki-67 antibody. Cells with a brown nucleus were judged to be Ki67-positive. The (HPF) count of 1000 tumor cells was randomly read by two pathologists from 10 highpower visual fields, and the Ki-67 proliferation index was calculated as the number of positive cells divided by the total cell count. The average value of the two physicians' readings was taken as the Ki- 67 proliferation index.

\section{Statistical analysis}

SPSS 25.0 statistical software was used to analyze the results, and Graphpad Prism 8.0 was used to draw graphs. The measurement data of normal distribution are expressed as absolute $\nabla x \pm S$, the differences between groups were compared by t-test, the measurement data of skewness distribution are expressed by $\mathrm{M}$ (range), and the comparisons between groups were calculated by Mann-Whitney U test. Categorical data are expressed as a percentage, and the Fisher exact probability was used for comparison between groups. Receiver operating characteristic curve analysis was used to evaluate the accuracy of the MRI quantitative data in distinguishing grade II SFT/HPC from grade III SFT/HPC, and the sensitivity and specificity of each parameter were calculated when the Youden index reached the maximum. The correlation of the MeanADC, MinADC, rADC, with the Ki-67 proliferation index was analyzed by Pearson correlation analysis. $P<.05$ indicated a statistically significant difference.

\section{Results}

\section{Patient characteristics}

In this study, there were 15 patients with grade II intracranial SFT/HPC and 22 with grade III intracranial SFT/HPC. There were 17 men and 20 women, with an average age of $51.32( \pm 12.06)$ years. Table 1 shows the clinical data of the patients. There was no significant difference in sex, age, or tumor size between patients with grade II and grade III SFT/HPC. 
Table 1

shows the clinical data of the patients.

\begin{tabular}{|c|c|c|c|c|c|}
\hline \multirow[t]{2}{*}{ Group } & & \multicolumn{2}{|l|}{ SFT/HPC } & \multirow{2}{*}{$x^{2} / t$} & \multirow[t]{2}{*}{$P$} \\
\hline & & II Grade & III Grade & & \\
\hline \multirow[t]{2}{*}{ Gender } & Male & $7(18.92 \%)$ & $10(27.03 \%)$ & \multirow[t]{2}{*}{-} & \multirow[t]{2}{*}{1} \\
\hline & Female & $8(21.62 \%)$ & $12(32.43 \%)$ & & \\
\hline \multirow[t]{3}{*}{ Location } & convex surface & $10(27.03 \%)$ & $13(35.14 \%)$ & \multirow[t]{3}{*}{0.227} & \multirow[t]{3}{*}{0.893} \\
\hline & tentorium & $4(10.81 \%)$ & $7(18.92 \%)$ & & \\
\hline & skull base & $1(2.7 \%)$ & $2(5.4 \%)$ & & \\
\hline \multicolumn{2}{|c|}{ Ki-67 proliferation index } & $10.67 \pm 4.49$ & $21.36 \pm 11.07$ & -4.065 & $<0.001$ \\
\hline \multicolumn{2}{|l|}{ Age } & $49.40 \pm 11.11$ & $52.64 \pm 13.02$ & -0.786 & 0.437 \\
\hline \multicolumn{2}{|c|}{ Tumor diameter } & $4.93 \pm 1.75$ & $4.72 \pm 1.56$ & 0.371 & 0.713 \\
\hline \multicolumn{2}{|c|}{ Tumor volume } & $54.79 \pm 41.79$ & $61.54 \pm 24.98$ & -0.615 & 0.542 \\
\hline
\end{tabular}

\section{Ki-67 proliferation index of grade II and grade III SFT/HPC}

The Ki-67 proliferative index in grade II SFT/HPC tumors was 10.67\% ( $\pm 4.49 \%)$, and the Ki-67 proliferative index in grade III SFT/HPC tumors was $21.36 \%$ ( $\pm 11.07 \%)$. The difference was statistically significant $(P$ $<.001$ ) (Table 1, Fig. 1).

\section{Correlation analysis of the intracranial SFT/HPC ADC values and the Ki-67 proliferation index}

The mean Ki-67 proliferation index of the 37 SFT/HPC tumors in this study was $17.03 \%( \pm 10.24 \%)$.

Pearson correlation analysis found that the MeanADC $(r=-0.427, P=.008)$, MinADC $(r=-0.356, P=.03)$, and $\operatorname{rADC}(r=-0.529, \mathrm{P}<.001)$ were negatively correlated with the Ki-67 proliferation index (Table 2, Fig. $1)$. 
Table 2

The mean Ki-67 proliferation index of the 37 SFT/HPC tumors in this study was $17.03 \%$ ( $\pm 10.24 \%)$. Pearson correlation analysis found that the MeanADC $(r=-0.427, P=.008)$, MinADC $(r=-0.356, P=.03)$, and $\operatorname{rADC}(r=$ $-0.529, \mathrm{P}<.001)$ were negatively correlated with the Ki-67 proliferation index

\begin{tabular}{|lll|}
\hline Parameters $\left(\times 10^{-3} \mathrm{~mm}^{2} / \mathbf{s}\right)$ & \multicolumn{2}{l|}{ Ki-67 proliferation index $(17.03 \pm 10.24) \%$} \\
\cline { 2 - 3 } & $\mathbf{r}$ & $\boldsymbol{p}$ \\
\hline MeanADC & -0.427 & 0.0083 \\
\hline MinADC & -0.356 & 0.0303 \\
rADC & -0.529 & 0.0007 \\
\hline
\end{tabular}

\section{Comparison of ADC values of grade II and grade III intracranial SFT/HPC}

There were significant differences in the MeanADC $(t=2.702, P=.011)$, MinADC $(t=2.730, P=.01)$, and $\operatorname{rADC}(\mathrm{t}=8.102, \mathrm{P}<.0001)$ between grade II and grade III SFT/HPC (Table 3). Representative cases of grade II and III SFT/HPC are shown in Fig. 2 and Fig. 3. Receiver operating characteristic curve analysis was used to determine the optimal cutoffs of the MeanADC, MinADC, and rADC for differentiating grade II and grade III SFT/HPC. The optimal cutoff of the MeanADC was $0.699 \times 10^{-3} \mathrm{~mm}^{2} / \mathrm{s}$, and the differential sensitivity and specificity were $93.3 \%$ and $50 \%$, respectively. The optimal cutoff of the MinADC was 0.645 $\times 10^{-3} \mathrm{~mm}^{2} / \mathrm{s}$, and the differential sensitivity and specificity were $93.3 \%$ and $63.6 \%$, respectively. The optimal cutoff of the rADC was 0.998 , and the differential sensitivity and specificity were $80 \%$ and $95.5 \%$, respectively (Fig. 4).

Table 3

There were significant differences in the MeanADC $(\mathrm{t}=2.702, \mathrm{P}=.011)$, MinADC $(\mathrm{t}$ $=2.730, \mathrm{P}=.01)$, and $\operatorname{rADC}(\mathrm{t}=8.102, \mathrm{P}<.0001)$ between grade $\mathrm{II}$ and grade III $\mathrm{SFT} / \mathrm{HPC}$

\begin{tabular}{|lllll|}
\hline Parameters $\left(\times 10^{-3} \mathrm{~mm}^{2} / \mathbf{s}\right)$ & SFT/HPC & \multicolumn{1}{l|}{ t } & $P$ \\
\cline { 2 - 5 } & II Grade & III Grade & & \\
\hline MaxADC & $0.853 \pm 0.098$ & $0.789 \pm 0.126$ & 1.632 & 0.112 \\
\hline MeanADC & $0.787 \pm 0.078$ & $0.693 \pm 0.119$ & 2.702 & 0.011 \\
\hline MinADC & $0.719 \pm 0.079$ & $0.622 \pm 0.121$ & 2.730 & 0.009 \\
\hline rADC & $1.078 \pm 0.126$ & $0.912 \pm 0.074$ & 8.102 & $<0.001$ \\
\hline
\end{tabular}




\section{Discussion}

Intracranial SFT/HPC originates from Zimmerman cells in meningeal interstitial capillaries and accounts for about $1 \%$ of intracranial tumors [12, 21]. The 2016 World Health Organization classification of CNS tumors states that the pathological diagnostic criteria for grade intracranial III SFT/HPC is mitotic activity of at least 5/10 HPF, tumor necrosis, hemorrhage, and moderate to high cell density [1]. This means that the Ki-67 proliferation index of grade III SFT/HPC tumor entities is often $\geq 10 \%[16,22,23]$. Furthermore, compared with grade II intracranial SFT/HPC, grade III SFT/HPC is associated with high postoperative recurrence, high metastasis, and low overall survival $[4,14,24]$. If the SFT/HPC grading can be clear before surgery, we can more accurately predict clinical postoperative tumor progression, which would allow surgeons to formulate more detailed treatment plans, including preoperative tumor interventions $[25,26]$, greater resection of the surrounding structure [4], and postoperative radiotherapy [27, 2]. However, it is still difficult for radiologists to effectively distinguish between grade II and grade III SFT/HPC before surgery. Previous studies have suggested that grade III SFT/HPC has certain MRI features, such as a narrow basement attached to the dura mater, increased necrosis/cystic degeneration, brain tissue edema, and heterogeneous enhancement $[16,17]$. However, these features are subjective. In addition, these semantic features cannot be used to quantitatively analyze changes in the microstructure of intracranial SFT/HPC tumor entities. Therefore, more objective, quantitative parameters are still needed to effectively distinguish these two tumor grades, so as to effectively guide the implementation of clinical treatment measures.

To the best of our knowledge, this study is the first to focus on the use of the ADC for graded diagnosis of SFT/HPC. DWI can non-invasively evaluate the Brownian motion of water molecules in the tissue. The $A D C$ is derived from DWI results and is mainly affected by the combined effect of the volume fractions inside and outside the cell. Therefore, the ADC can be of great significance for tumor grading, preoperative quantitative assessment, and differential diagnosis of brain tumors [3]. This study analyzed the ADCs of grade II and grade III SFT/HPC tumor entities and found that the MinADC, MeanADC, and rADC were significantly different in grade II and grade III SFT/HPC. The IADC had the highest accuracy in distinguishing grade II and grade III SFT/HPC (area under the curve $=0.897$ ), with a diagnostic sensitivity and specificity of $80 \%$ and $95.5 \%$, respectively. Chen et al [5]. found that the standardized ADC is of great significance in distinguishing SFT/HPC from meningioma. When the threshold of the standardized ADC is greater than 1.15 , the sensitivity and specificity for distinguishing these two malignancies are $75 \%$ and $60.42 \%$, respectively. Although they did not perform subgroup analysis of grades II and III intracranial SFT/HPC, they concluded that the ADC was negatively correlated with tumor malignancy and positively correlated with the extracellular space. Tumor cells in grade III SFT/HPC have increased mitotic activity and an enlarged nucleus and cytoplasm, resulting in the shrinkage of the space around the cells. This restricts the diffusion of water molecules, which decreases the ADC $[3,6]$. Therefore, the ADC can reflect changes in the microstructure of tissues. Horvat et al [7]. found that the ADC of tumors can be used to differentiate molecular subtypes of invasive breast cancer. The maximum ADC has the highest diagnostic efficiency, which means that assessing the ADC before surgery can help doctors predict tumor grade and patient prognosis. The results of this study support the conclusions of previous studies 
showing that the ADC can non-invasively achieve tumor grading and typing, thereby assisting in the development of personalized treatment plans before surgery.

Histopathology can accurately distinguish grade II and III intracranial SFT/HPC based on tumor microstructure and cell morphology. The Ki-67 proliferation index is an important indicator that indirectly reflects the degree of tumor malignancy. Previous studies have shown that a higher Ki-67 proliferation index is correlated with an increased probability of tumor progression $[4,8,9]$. Multiple studies have shown that there is a correlation between the Ki-67 proliferation index of solid tumors and the ADC. Xianwang et al [10]. found a strong negative correlation between the MinADC and the Ki-67 proliferation index in ependymoma. He et al [20]. found that the MinADC and the Ki-67 proliferation index have a slight negative correlation in SFT/HPC, which is consistent with the results of this study. This negative correlation may be due to differences in cell cycle progression. Smaller cells are in G1 phase and larger cells are in $\mathrm{G} 2 / \mathrm{M}$ phase, and as the cell volume increases, the extracellular volume decreases and the ADC decreases. The Ki-67 proliferation index is related to cell proliferation, and high expression of $\mathrm{Ki}-67$ is observed in the G2/M phase. As the mitotic activity of the cell intensifies, the ADC gradually decreases and Ki-67 gradually increases, which leads to this negative correlation $[11,13]$. This study found that the MeanADC, MinADC, and $\mathrm{rADC}$ had a strong negative correlation with the Ki-67 proliferation index in SFT/HPC. Because this tumor microstructure causes macroscopic differences in the ADC, the preoperative ADC predicts the Ki-67 proliferation index of intracranial SFT/HPC tumors. Therefore, the $A D C$ is a preoperative, non-invasive factor that can assist surgeons in developing a detailed surgical plan and follow-up program.

This study had several limitations. First, this study took place in a single center and had a small sample size. In addition, because grade III SFT/HPC has more indicative radiological features than grade II SFT/HPC, such as necrosis, obvious cerebral edema, and heterogeneous enhancement, this may cause a certain subjective deviation in the manual measurement of the ADC. Finally, there is a lack of complete follow-up data, and the value of the ADC in the preoperative differentiation of grade II and III intracranial SFT/HPC needs further verification.

\section{Conclusions}

In summary, this study used the preoperative MeanADC, MinADC, and $\mathrm{rADC}$ to effectively distinguish grade II and III SFT/HPC tumor entities. The study also found that there is a strong negative correlation between the ADC and the tumor Ki-67 proliferation index. These conclusions will help clinicians formulate a personalized surgery plan for intracranial SFT/HPC.

\section{Declarations}

Funding: This study was supported by grants of National Natural Science Foundation of China (grant numbers of 81772006 and 82071872 ). 
Conflict of interest: The authors declare that they have no conflict of interest.

Availability of data and material: Not applicable.

Code availability: Not applicable.

Ethics approval : This study was approved by the Medical Ethics Committee of the Second Hospital of Lanzhou University and informed consent was waived.

Consent to participate: Thanks to the National Natural Science Foundation of China.

Consent for publication: Agree to publish.

\section{Authors' contributions】}

First author区Shenglin Li $₫$ Theoretical design, article writing, data processing $\mathbb{\bigotimes}$

Co-first author $\bigotimes$ Qing Zhou $\quad$ TTheoretical design, article writing, data processing $\mathbb{\bigotimes}$

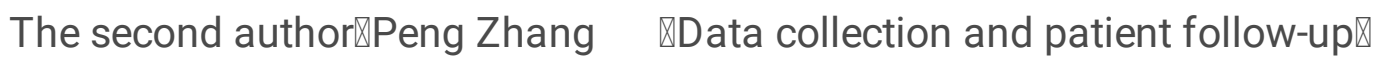

The third author $\$ Shize $\mathrm{Ma} \quad$ $\quad$ Theoretical guidance and patient follow-up $\rrbracket$

The fourth author: Caiqiang Xue 『data processing $\rrbracket$

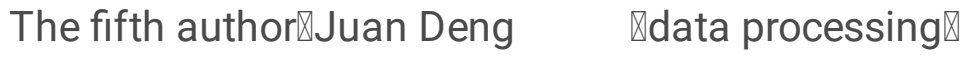

The sixth author $\llbracket$ Xianwang Liu ¿data processing $\rrbracket$

Corresponding author: Junlin Zhou, MD, PhD. $₫$ Theoretical guidance and article revision suggestions $\rrbracket$

\section{References}

1. Louis DN, Perry A, Reifenberger G, von Deimling A, Figarella-Branger D, Cavenee WK et al (2016) The 2016 World Health Organization Classification of Tumors of the Central Nervous System: a summary. Acta Neuropathol 131(6):803-820. https://doi.org/10.1007/s00401-016-1545-1

2. Cohen-Inbar O, Lee CC, Mousavi SH, Kano H, Mathieu D, Meola A et al (2017) Stereotactic radiosurgery for intracranial hemangiopericytomas: a multicenter study. J Neurosurg 126(3):744754. https://doi.org/10.3171/2016.1.JNS152860

3. Yamasaki F, Kurisu K, Satoh K, Arita K, Sugiyama K, Ohtaki M et al (2005) Apparent diffusion coefficient of human brain tumors at MR imaging. Radiology 235(3):985-991. https://doi.org/10.1148/radiol.2353031338

4. Ratneswaren T, Hogg FRA, Gallagher MJ, Ashkan K (2018) Surveillance for metastatic hemangiopericytoma-solitary fibrous tumors-systematic literature review on incidence, predictors and 
diagnosis of extra-cranial disease. J Neurooncol 138(3):447-467. https://doi.org/10.1007/s11060018-2836-2

5. Chen T, Jiang B, Zheng Y, She D, Zhang H, Xing Z et al (2020) Differentiating intracranial solitary fibrous tumor/hemangiopericytoma from meningioma using diffusion-weighted imaging and susceptibility-weighted imaging. Neuroradiology 62(2):175-184. https://doi.org/10.1007/s00234019-02307-9

6. Nagar VA, Ye JR, Ng WH, Chan YH, Hui F, Lee CK et al (2008) Diffusion-weighted MR imaging: diagnosing atypical or malignant meningiomas and detecting tumor dedifferentiation. AJNR Am J Neuroradiol 29(6):1147-1152. https://doi.org/10.3174/ajnr.A0996

7. Horvat JV, Bernard-Davila B, Helbich TH, Zhang M, Morris EA, Thakur SB et al (2019) Diffusionweighted imaging (DWI) with apparent diffusion coefficient (ADC) mapping as a quantitative imaging biomarker for prediction of immunohistochemical receptor status, proliferation rate, and molecular subtypes of breast cancer. J Magn Reson Imaging 50(3):836-846. https://doi.org/10.1002/jmri.26697

8. Spatola C, Privitera G (2004) Recurrent intracranial hemangiopericytoma with extracranial and unusual multiple metastases: case report and review of the literature. Tumori 90(2):265-268

9. Demirtas E, Ersahin Y, Yilmaz F, Mutluer S, Veral A (2000) Intracranial meningeal tumours in childhood: a clinicopathologic study including MIB-1 immunohistochemistry. Pathol Res Pract 196(3):151-158. https://doi.org/10.1016/S0344-0338(00)80095-3

10. Xianwang L, Lei H, Hong L, Juan D, Shenglin L, Caiqiang X et al (2020) Apparent Diffusion Coefficient to Evaluate Adult Intracranial Ependymomas: Relationship to Ki-67 Proliferation Index. J Neuroimaging 31(1):132-136. https://doi.org/10.1111/jon.12789

11. Huang Z, Xu X, Meng X, Hou Z, Liu F, Hua Q et al (2015) Correlations between ADC values and molecular markers of Ki-67 and HIF-1alpha in hepatocellular carcinoma. Eur J Radiol 84(12):24642469. https://doi.org/10.1016/j.ejrad.2015.09.013

12. Stout AP, Murray MR (1942) Hemangiopericytoma: A Vascular Tumor Featuring Zimmermann's Pericytes. Ann Surg 116(1):26-33. https://doi.org/10.1097/00000658-194207000-00004

13. Li X, Jiang H, Niu J, Zheng Y (2014) Correlation of ADC value with pathologic indexes in colorectal tumor homografts in Balb/c mouse. Chin J Cancer Res 26(4):444-450. https://doi.org/10.3978/j.issn.1000-9604.2014.08.06

14. Rutkowski MJ, Jian BJ, Bloch O, Chen C, Sughrue ME, Tihan T et al (2012) Intracranial hemangiopericytoma: clinical experience and treatment considerations in a modern series of 40 adult patients. Cancer 118(6):1628-1636. https://doi.org/10.1002/cncr.26411

15. Damodaran O, Robbins P, Knuckey N, Bynevelt M, Wong G, Lee G (2014) Primary intracranial haemangiopericytoma: comparison of survival outcomes and metastatic potential in WHO grade II and III variants. J Clin Neurosci 21(8):1310-1314. https://doi.org/10.1016/j.jocn.2013.11.026

16. Zhou JL, Liu JL, Zhang J, Zhang M (2012) Thirty-nine cases of intracranial hemangiopericytoma and anaplastic hemangiopericytoma: a retrospective review of MRI features and pathological findings. 
Eur J Radiol 81(11):3504-3510. https://doi.org/10.1016/j.ejrad.2012.04.034

17. Chiechi MV, Smirniotopoulos JG, Mena H (1996) Intracranial hemangiopericytomas: MR and CT features. AJNR Am J Neuroradiol 17(7):1365-1371

18. Mama N, Ben Abdallah A, Hasni I, Kadri K, Arifa N, Ladib M et al (2014) MR imaging of intracranial hemangiopericytomas. J Neuroradiol 41(5):296-306. https://doi.org/10.1016/j.neurad.2013.10.007

19. Onishi N, Kanao S, Kataoka M, lima M, Sakaguchi R, Kawai M et al (2015) Apparent Diffusion Coefficient as a Potential Surrogate Marker for Ki-67 Index in Mucinous Breast Carcinoma. J Magn Reson Imaging 41(3):610-615. https://doi.org/10.1002/jmri.24615

20. He W, Xiao X, Li X, Guo Y, Guo L, Liu X et al (2019) Whole-tumor histogram analysis of apparent diffusion coefficient in differentiating intracranial solitary fibrous tumor/hemangiopericytoma from angiomatous meningioma. Eur J Radiol 112:186-191. https://doi.org/10.1016/j.ejrad.2019.01.023

21. Soyuer S, Chang EL, Selek U, McCutcheon IE, Maor MH (2004) Intracranial meningeal hemangiopericytoma: the role of radiotherapy: report of 29 cases and review of the literature. Cancer 100(7):1491-11497. https://doi.org/10.1002/cncr.20109

22. Demaerel P, Wilms G, Lammens M, Marchal G, Plets C, Goffin J et al (1991) Intracranial meningiomas: correlation between MR imaging and histology in fifty patients. J Comput Assist Tomogr 15(1):45-51

23. Yamamoto Y, Hayashi Y, Murakami I (2020) Recurrence of Solitary Fibrous Tumor/Hemangiopericytoma Could Be Predicted by Ki-67 Regardless of Its Origin. Acta Med Okayama 74(4):335-343. https://doi.org/10.18926/AMO/60372

24. Melone AG, D'Elia A, Santoro F, Salvati M, Delfini R, Cantore G et al (2014) Intracranial hemangiopericytoma-our experience in 30 years: a series of 43 cases and review of the literature. World Neurosurg 81(3-4):556-562. https://doi.org/10.1016/j.wneu.2013.11.009

25. Uemura S, Kuratsu J, Hamada J, Yoshioka S, Kochi M, Ushio Y et al (1992) Effect of radiation therapy against intracranial hemangiopericytoma. Neurol Med Chir (Tokyo) 32(6):328-332. https://doi.org/10.2176/nmc.32.328

26. lampreechakul P, Tirakotai W, Lertbutsayanukul P, Siriwimonmas S, Liengudom A (2016) Preoperative Embolization of Intracranial and Extracranial Tumors: A Review of 37 Cases. J Med Assoc Thai 99(Suppl 3):S91-S119

27. Patel AR, Flores BC, Ban VS, Hatanpaa KJ, Mickey BE, Barnett SL (2017) Intracranial Hemangiopericytomas: Recurrence, Metastasis, and Radiotherapy. J Neurol Surg B Skull Base 78(4):324-330. https://doi.org/10.1055/s-0037-1599073

\section{Figures}



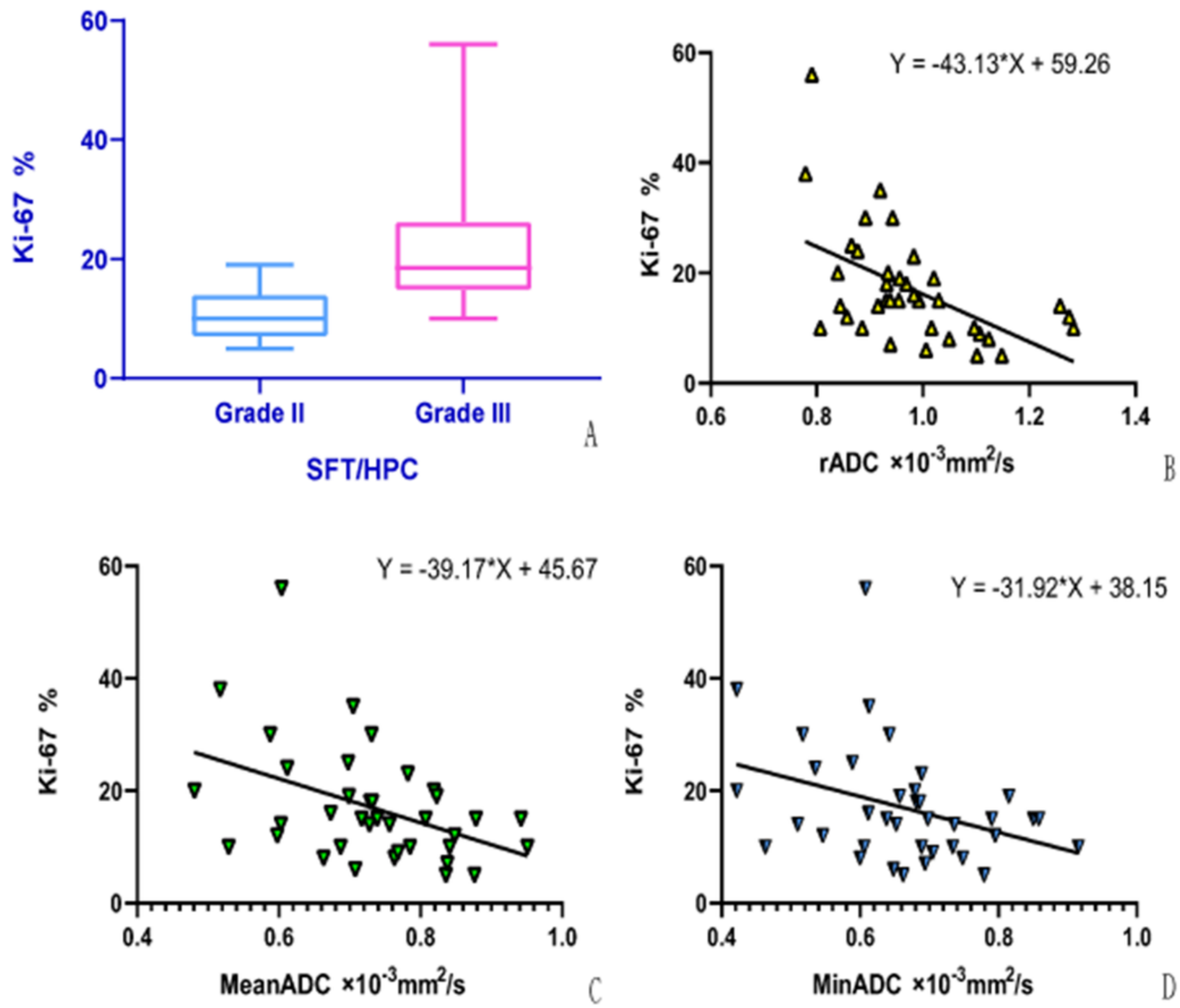

Figure 1

A: Ki-67 proliferation indexes of grade II and grade III SFT/HPC. B: The relative (B), average (C) and minimum (D) ADC and Ki-67 were negatively correlated. SFT/HPC: solitary fibrous tumor/hemangiopericytoma 


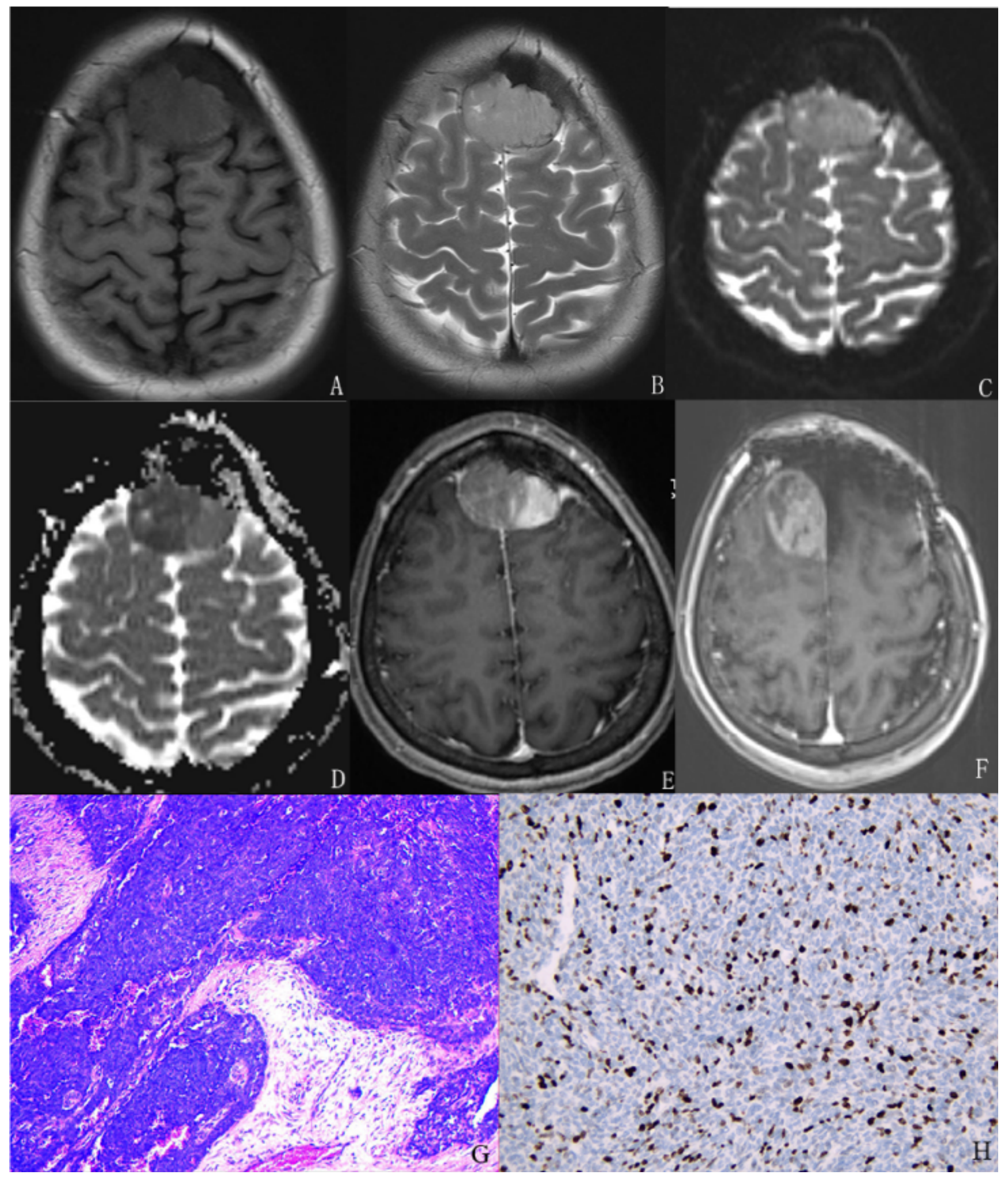

Figure 2

A 38-year-old woman with grade III SFT/HPC in the frontal region. A-E: T1WI and T2WI were relatively uniform before operation, and the frontal bone cancellous signal was abnormal. The DWI and ADC indicated that the tumor was restricted in spreading and the enhancement was uneven. F: Re-examination of the head 8 months after surgery revealed that the right frontal area was round-shaped with uneven 
enhancement. G-H: Pathology $(H \& E \times 100)$ showed a large number of heterogeneous cells in the mirror image, and the Ki-67 index was about $30 \%$.

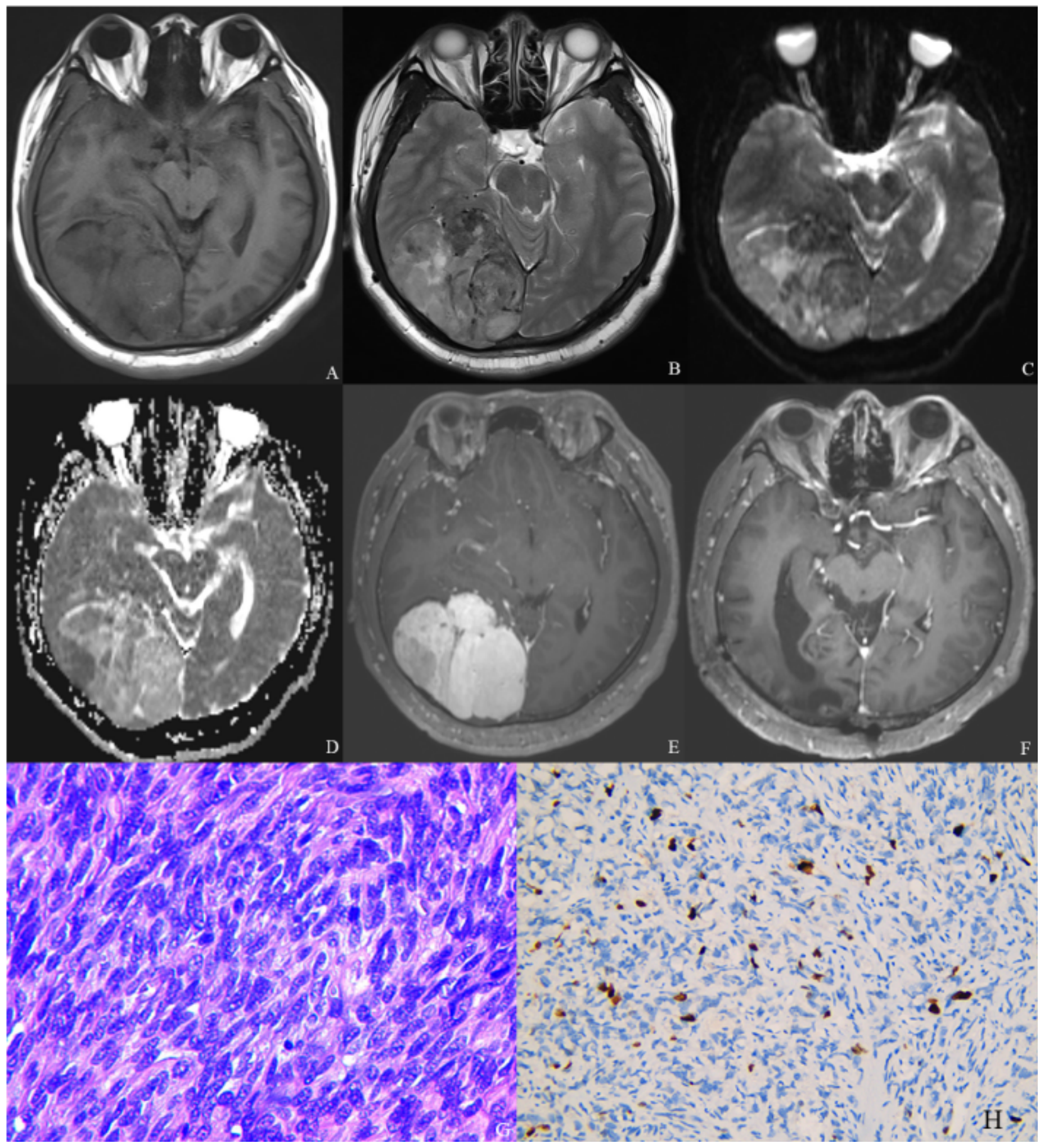

\section{Figure 3}

A 54-year-old man with a round grade II HPC in the right temporo-occipital region. A-E: Tumor T1WI and T2WI signals were uneven, whereas DWI showed an uneven and slightly high signal. The ADC was uneven and low, the tumor-brain interface was clear, and the enhancement was obvious. F: Thirty-six 
months after the operation, head MRI showed the formation of softening of the brain area, and no tumor recurrence was seen. G-H: Pathological map of intracranial HPC (H\&E ×200), and the Ki-67 index was 8\%.

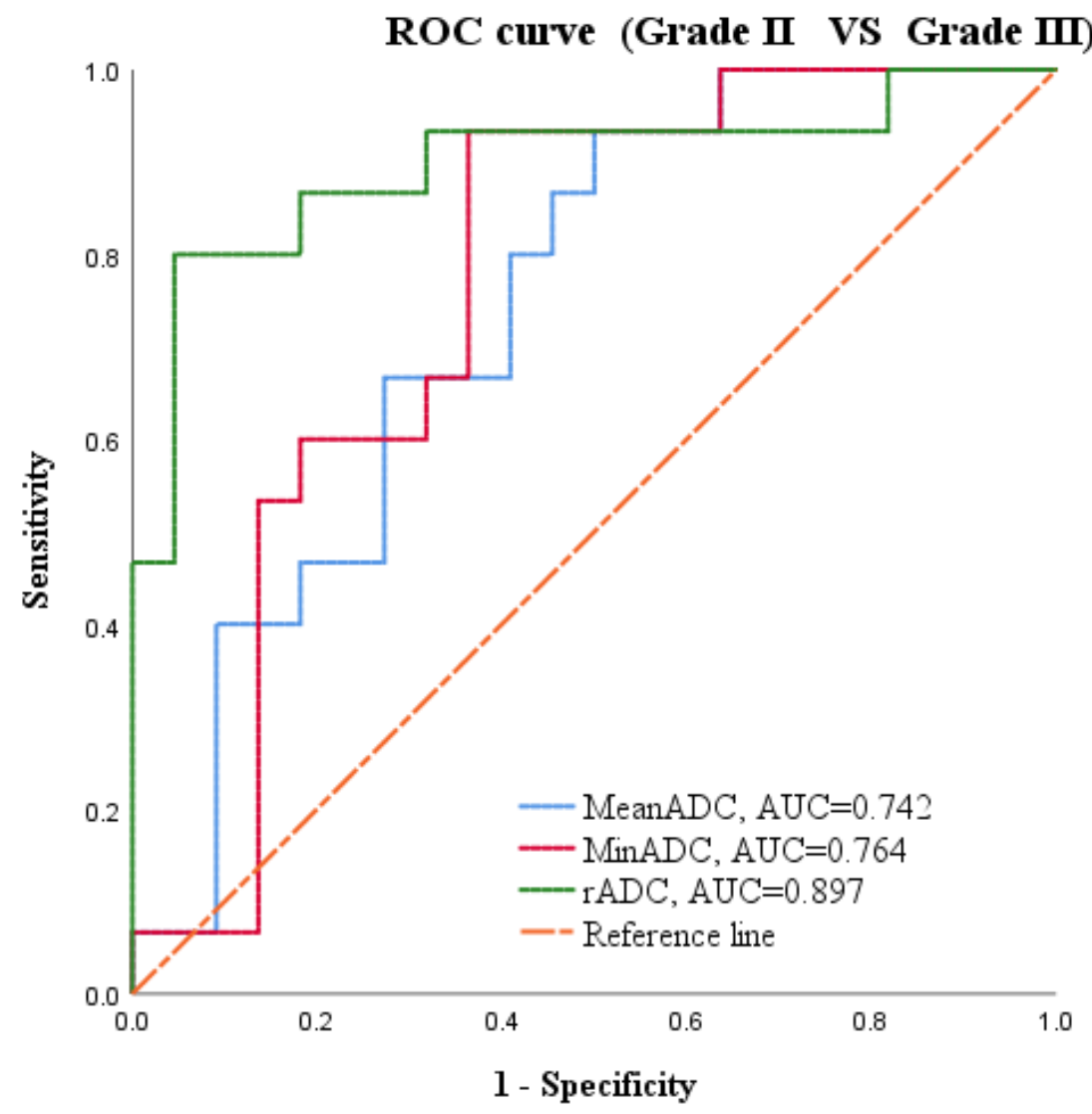

\section{Figure 4}

Receiver operating characteristic curve analysis of the average, minimum, and relative ADC in differentiating grade II and grade III SFT/HPC. SFT/HPC: solitary fibrous tumor/hemangiopericytoma 